\title{
Role of HIIT VS High Intensity Cardio in Fat Reduction
}

\author{
Yaseen $A^{*}$ \\ Department of physiotherapy, DOW University of Health Sciences, Pakistan
}

*Corresponding author: Amna Yaseen, Department of physiotherapy, DOW

University of Health Sciences, Pakistan; Email: amna_yaseen2011@yahoo.com

\author{
Editorial \\ Volume 2 Issue 3
}

Received Date: July 29, 2019

Published Date: August 23, 2019

DOI: $10.23880 /$ aphot- 16000137
Abbreviations: HIIT: High intermittent interval training; HIPT: high intensity power training.

WHO entitled overweight and obesity as an inappropriate and extra accretion of fats in the body that can displays a hazard to wellbeing. A crude proportion of obesity is BMI (weight $(\mathrm{kg}) /$ height $\left(\mathrm{m}^{2}\right)$. A person having BMI $>30$ is typically consider as obese and person $\mathrm{BMI} \leq 25$ is labeled as overweight [1]. Putting on weight and being fat is a problem of public health concern. Epidemiological studies showed that majority includes population who are aged between 20 to 40 years. WHO reported in 2016 world's $13 \%$ adult population is obese $(11 \%$ males and $15 \%$ females) drastically level of it is continuously increasing in both developed and developing countries [2]. Poorly overweight and obesity lowers the quality of life. The global epidemic of overweight and obesity prone the population towards the cardiovascular disorders, metabolic disorders, cancers and hormonal problems. The most common reason behind this epidemic disorder is lack of physical activity and the luxurious lifestyle [3].

Habitué exercises have a great effect on the body. It's an economically executable, cost effective and nonpharmacological intervention to reduce a health hazard [4]. It is use as a primary intervention that delays and in many cases prevents the health burdens associated with several chronic diseases. However, the specific regimen of workout is needed to accrue health benefits. Whether the workout is done for weight loss or to live a healthy life, either endurance training, aerobics, pyramids, cardio or high intermittent interval training (HIIT) is performed, every strategy influences the mechanism of the body by increasing the maximum volume of oxygen, decreasing the lipids, decreasing the risk of cardiovascular disorders and promote a sense of well-being [5]. For healthy living moderate-intensity continuous exercise program is typically suggested to adults for the improvement and maintenance of physical health and fitness as they are safe and keeps the individual healthy and fit. Program regimen includes $30 \mathrm{mins} /$ day, 5 days/week or a total workout of $150 \mathrm{mins} /$ week or alternatively high intensity of workout for 75mins/week [6]. Poorly, these kinds of regimens resulted in minimum fat loss [5].

Comparatively, evidence suggested that high intensity interval training (HIIT) is an effective strategy for fat loss Though there isn't any comprehensive definition of High intensity training (HIT) or high intermittent training (HIT) or (HIIT) but usually refers to repeated sessions of relatively transient intermittent exercise, that is performed with "all-out" effort or intensity which evoke V02peak (i.e., Q90\% of V02peak) [1]. Depending on the work intensity, one effort may last from variety of seconds up to several minutes, with multiple efforts separated by up to variety of minutes of rest or lowintensity exercise [7]. High intensity interval work involves repeatedly travail at a high intensity for thirty seconds to several minutes, separated by 1-5 minutes of recovery (either no or low intensity exercise). HIT is expounded to raised patient compliance and improved vessel and metabolic outcomes and is suitable for implementation in every healthy and 'at risk' populations. HIT exercises includes like running, cycling athletics activities. More importantly it doesn't include the activity that cause muscle fibers hypertrophy. A typical HIT intervention model is that the Wingate check, that involves thirties of complete greatest athletics against a high braking force on a specialized instrument [7]. A relatively new variation of HIIT has recently become common and that incorporates high-intensity resistance training that involves, multiple-joint movements and is known as high intensity power training (HIPT). It is becoming common all over the world because it's enhance the aerobic fitness with in minimum duration, despite controversy is present over it [8]. 


\section{Annals of Physiotherapy \& Occupational Therapy}

Evidence showed that HIT training provides tremendous benefits in a very short time period because it incorporates continual bursts of intense exercise sprinkled with low power recuperation. There is developing verification from sound populaces that HIIT at last winds up amidst type of vessel and metabolic advantages, improvement of endocrine sensitivity, organic process fitness, effective in fat loss, positive effects on cardio metabolism, reduces the risk of diabetes, reduces the risk of hypertension thus by decreasing lipoprotein cholesterol level and therefore in the long term it maintains the blood pressure and prevent from associated comorbidities [9-12]. Thus, in spite of all unelectable benefits of HIIT there are still questions arises that is it beneficial for every age group of population? Are the exercise protocols are same and safe for everyone? Why the effects of HIT are unclear in the case of insulin sensitivity? Is the HIT regimen is safe for the people who are not just obese but also having blood coagulopathies.

As compare to cardio training, high intermittent training have efficient results in a shorter period of time therefore physical trainers just promotes it?

\section{References}

1. Trapp EG, Chisholm DJ, Freund J (2008) The effects of high-intensity intermittent exercise training on fat loss and fasting insulin levels of young women. Int J Obes 32(4): 684-691.

2. Heydari M, Freund J, Boutcher SH (2012) The Effect of High-Intensity Intermittent Exercise on Body Composition of Overweight Young Males. Journal of Obesity, pp: 1-8.

3. Keating SE, Machan EA, O'connor HT (2014) Continuous Exercise but Not High Intensity Interval Training Improves Fat Distribution in Overweight Adults. J Obes (2014): 1-12.

4. Irving BA, Davis CK, Brock DW (2008) Effect of exercise training intensity on abdominal visceral fat and body composition. Med Sci Sports Exerc 40(11): 1863-1872.

5. Paoli A, Paoli QF, Moro T (2013) Effects of highintensity circuit training, low-intensity circuit training and endurance training on blood pressure and lipoproteins in middle-aged overweight men. Lipids Health Dis 3(12): 131.

6. Gormley SE, Swain DP, High R (2008) Effect of intensity of aerobic training on VO2max. Med Sci Sports Exerc 40(7): 1336-1343.

7. Gibala MJ, Mcgee SL (2008) Metabolic Adaptations to Short-term High-Intensity Interval Training: A Little Pain for a Lot of Gain? Exerc Sport Sci Rev 36(2): 5863.

8. Smith MM, Sommer AJ, Starkoff BE (2013) Crossfitbased high-intensity power training improves maximal aerobic fitness and body composition. J Strength Cond Res 27(11): 3159-3172.

9. Sosner P, Gayda M, Drigny J (2013) Effects of longterm lifestyle and high-intensity interval training intervention on blood pressure reduction in patients with abdominal obesity. European heart journal 34(1): 659 .

10. Helgerud J, Hoydal K, Wang E (2007) Aerobic highintensity intervals improve V02max more than moderate training. Med Sci Sports Exerc 39(4): 665671.

11. Gormley SE, Swain DP, High R (2008) Effect of intensity of aerobic training on VO2max. Med Sci Sports Exerc 40(7): 1336-1343.

12. Gibala MJ, Little JP, Macdonald MJ (2012) Physiological adaptations to low-volume, high-intensity interval training in health and disease. J physiol 590(5): 1077-1084. 